\title{
Patient Safety and Unplanned Extubation in a Pediatric Intensive Care Unit: A Analytical Observational Study
}

\author{
Ribeiro ER ${ }^{1 *}$, Pellis IC ${ }^{2}$, Maranhao $\mathrm{JGW}^{2}$, Gennari $\mathrm{RL}^{2}$, Simao $\mathrm{M}^{3}$ and \\ Pamplona $\mathrm{RAC}^{3}$ \\ ${ }^{1}$ Professor and researcher in the Health Sciences Teaching Program and medical course at \\ Faculdades Pequeno Príncipe, Brazil \\ ${ }^{2}$ Academics from Medicine course at Faculdades Pequeno Príncipe, Brazil \\ ${ }^{3}$ Master's degree in the Health Sciences Teaching Program at Faculdades Pequeno Príncipe, \\ Brazil
}

\section{Research Article}

Volume 4 Issue 2

Received Date: March 08, 2021

Published Date: March 24, 2021

DOI: $10.23880 /$ jqhe-16000211

*Corresponding author: Elaine Rossi Ribeiro, Professor and researcher in the Health Sciences Teaching Program and medical course at Faculdades Pequeno Príncipe, Brazil; Email: elaine.rossi@hotmail.com

\section{Abstract}

Introduction: Unplanned extubation (UE) is characterized by the removal or displacement of the endotracheal tube (ETT). Objectives: To analyze the incidence of unplanned extubation, characterize the most prevalent cases of unplanned extubation and analyze an extubation protocol.

Methodology: This study is characterized as an analytical observational study design, performed in two stages: field research to collect and analyze data from medical records and analysis of the NPE protocol used by a large hospital.

Results: In the collected medical records, rates of 7.75 UE/100 days of MV for general and surgical ICU and 4.68 UE/100 days of MV for cardiopediatric ICU were found. The female gender was predominant in the group of patients evaluated and the cause of unknown origin was the most prevalent. We identified 19 unplanned extubations in the general and surgical ICU, and 9 episodes of unplanned extubation in the cardiopediatric ICU, 28 occurrences. For the protocol evaluation the AGREE II instrument was used and the following scores were obtained: domain 1 with 85.19\%; domain 2 with 72.22\%; domain 3 with $35.42 \%$; domain 4 with $96.30 \%$; domain 5 with $76.39 \%$ and domain 6 with $100 \%$. The general score of the protocol evaluation was grade four.

Conclusion: The data presented can be of great benefit for prevention, identification and early intervention of UE episodes in pediatric patients with higher risk factors.

Keywords: Extubation; Patient Safety; Intensive Care Unit; Pediatric

Abbreviations: UE: Unplanned Extubation; ETT: Endotracheal Tube; PICU: Pediatric Intensive Care Unit; OTI: Orotracheal Intubation; MV: Mechanical Ventilation; AE: Adverse Events; VAP: Ventilator Associated Pneumonia; NICU: Neonatal Intensive Care Units; ICU: Intensive Care Unit; AGREE: Appraisal of Guidelines for Research \& Evaluation;
OCT: Orotracheal Cannula; NOC: Clinical Guidance Standards.

\section{Introduction}

Patient safety in the Pediatric Intensive Care Unit (PICU) portrays a challenging scenario for physicians. The 
resoluteness of cases and the adoption of procedures that minimize adverse events, as well as favorable outcomes, are factors that make up their routine. Among these procedures, the use of orotracheal intubation (OTI) technique is a procedure repeatedly performed in PICUs.

Tracheal intubation can be defined as the placement of a tube into the trachea, either orally or nasally [1]. The endotracheal tube (ETT) can be removed electively when the cause of mechanical ventilation (MV) has been resolved.

However, unplanned - or accidental - extubation is considered a recurrent and commonly avoidable adverse event [2]. Adverse events (AE) are defined as unwanted complications resulting from patient care that are not attributed to the natural evolution of the underlying disease [3].

Unplanned extubation is defined as any extubation that is unexpected or performed at an unscheduled time due to patient agitation or caregiver handling [4].

Unplanned extubations usually lead to emergent and less controlled reintubation [5]. Repeated intubations can cause airway (larynx or trachea) and lung injuries and scarring, favoring the incidence and occurrence of VAP (ventilatorassociated pneumonia) [5].

Unplanned extubations are associated with worsening factors in the clinical picture, such as increased length of hospital stay, risk of infections, increased days on mechanical ventilation, in addition to cardiopulmonary resuscitation and use of resuscitation medications [6].

For comparison, the unplanned extubation rates should present the ratio of the number of unscheduled extubations per 100 day-intubated patients [7]. This incidence rate includes the factor of days at risk of extubation and allows comparisons between different neonatal intensive care units (NICU) [7].

This study demonstrated that unplanned extubation in the pediatric population has serious consequences, among them prolonged mechanical ventilation, pediatric ICU, hospital admissions and increased costs, but there is no association with increased mortality [8].

In the newborn age group, there is a greater threat of unplanned extubation [7]. It is because at this age there is difficult fixation of the tube on the small surface of the face, the short length of the trachea and the use of endotracheal tubes without a balloon [7].
Given the importance of this potentially preventable adverse effect, unplanned extubation rate has been used as one of the indicators in the quality of service assessments [4].

Given this scenario, the research question was established: what is the incidence of unplanned extubation and its associated risk factors? To answer this question, the objectives of this study were to analyze the incidence of unplanned extubation, and as a specific objective, to analyze the extubation protocol of a large pediatric hospital in Curitiba-PR, Brazil.

\section{Methods}

This study is characterized as an analytical observational study design, with a quantitative approach, performed in two stages. The research field was a large pediatric hospital located in the city of Curitiba-PR. In the first stage, a documentary analysis of medical records was performed on the indicators of unplanned extubation in the cardiac, general, and surgical Intensive Care Units (ICU) of that hospital from January to December 2019. The set of information was analyzed by the researchers, with a thorough inspection of the patients' evolution records and collection of pertinent information for the research.

A sample of 22 patients was collected. The data collection instrument contained the following information: gender, patient's age, and need for reintubation, diagnosis, outcome and cause of extubation.

As inclusion criteria, only the medical records of patients admitted to the cardiopediatric, general, and surgical ICU of the hospital in question, in the period from January to December 2019, were analyzed. Those without a history of ICU evolution or with incomplete data were excluded.

Subsequently, in the second stage, the formalized protocol used by the hospital was verified, with the title "Prevention of unplanned extubation." The AGREE II (Appraisal of Guidelines for Research \& Evaluation) instrument was applied in this assessment. AGREE II is a tool that assesses the methodological rigor and transparency with which a NOC is developed [9].

\section{Results}

\section{Stage 1 - Analysis of Medical Records}

Based on the analysis of the medical charts, using simple descriptive statistics, the information collected about the patients who suffered unplanned extubation, and comparing 
the data from the cardiopediatric ICU with those from the general and surgical ICU, it was identified that among the analyzed period, 22 pediatric patients admitted to the ICU presented 01 or more episodes of unplanned extubation. The length of stay in ICU varied for each patient, taking a minimum of 9 and a maximum of 232 days. The length of stay intubated was another observed indicator, extending from a few minutes to hours associated with surgical procedures, lasting from days to weeks.

Regarding the diagnosis of patients admitted to the ICUs, the most common associated factor is congenital causes, corresponding to $100 \%$ of the diagnoses in the cardiopediatric ICU and $67 \%$ in the general and surgical ICU. Non congenital causes are responsible for about 33\% remaining in the general and surgical ICU.

The age range showed a difference in the two groups of patients studied. Around $53 \%(n=8)$ of the patients in the general and surgical ICU and $55 \%(n=6)$ in the cardiopediatric ICU were one year old or older.

Females were predominantin both groups, approximately $64 \%(n=7)$ in the general and surgical ICU and $55 \%(n=6)$ in the cardiopediatric ICU.

The need for reintubation is a parameter that expresses high rates of occurrence, being accounted for in $82 \%(n=9)$ of the general and surgical ICU patients and in $78 \%(n=7)$ of the cardiopediatric ICU patients.

The outcome of patients reported by the electronic medical records shows a prevalence of the non-death indicator. The data show that in the general and surgical ICU only $9 \%(n=1)$ of the cases resulted in death, while in the cardiopediatric ICU, there were deaths in $18 \%(n=2)$ of the cases, twice the percentage indicated in the cardiopediatric ICU.

The main causes of unplanned extubation described in the charts were divided into 4 groups: unknown cause, patient transfer/movement (orotracheal cannula manipulation)/ during the procedure, equipment disconnection, and patientrelated (agitation, secretion).

In the general and surgical ICU and cardiopediatrics $47 \%(n=7)$ and $30 \%(n=3)$ of the causes of unplanned extubation, respectively, denote unknown origin since they were not properly filled in the evolution sheets. Both samples exhibit $20 \%$ of the causes related to procedures, and transfer and manipulation of the OCT (orotracheal cannula). The disconnection of the equipment represented $7 \%(\mathrm{n}=1)$ in the general and surgical ICU patients and $30 \%(n=3)$ in the cardiopediatric ICU patients (Tables1\&2).

\begin{tabular}{|c|c|c|}
\hline Causes of unplanned extubation & General and Surgical ICU (\%) & Cardiopediatric ICU (\%) \\
\hline Unknown cause & 47 & 30 \\
\hline Patient transfer/movement & 20 & 20 \\
\hline Equipment disconnection & 7 & 30 \\
\hline Patient-related & 26 & 20 \\
\hline Total & $100 \%$ & $100 \%$ \\
\hline
\end{tabular}

Table 1: The main causes of unplanned extubation.

In total, 19 unplanned extubations in the general and surgical ICU and 9 episodes of unplanned extubation in the cardiopediatric ICU were identified by the authors, totaling 28 occurrences. However, this number does not represent the total of extubations, since one of the limiting factors of the study was the difficulty in data collection due to lack of information in the medical charts.

Incidence Analysis

The incidences of the medical records collected from 21 patients were analyzed. The numbers found were 7.75 UE/100 days of MV for general and surgical ICU and 4.68 UE/100 days of MV for cardiopediatric ICU.

\begin{tabular}{|c|c|}
\hline Incidences & UE/100 days of MV \\
\hline General and Surgical ICU & 7.75 \\
\hline Cardiopediatric ICU & 4.68 \\
\hline
\end{tabular}

Table 2: The incidences of the medical records collected from 21 patients were analyzed.

\section{Stage 2- Unplanned Extubation Prevention Protocol Analysis}

The Unplanned Extubation Prevention Protocol used by the hospital in which the research was conducted was analyzed using the AGREE II tool. 
The AGREE Instrument (Appraisal of Guidelines for Research \& Evaluation) was developed to address variability in the quality of a NOC (Clinical Guidance Standards [9].

The AGREE II is composed by 23 key items organized into 6 domains, followed by two global rating items ("global assessment"). Each domain captures a single quality dimension of the NOC. Each AGREE II item and the two global rating items are rated on a 7-point Likert scale (from 1-strongly disagree to 7-strongly agree) [9].

The instrument presents six domains. Domain 1: scope and purpose, domain 2: stakeholder involvement, domain 3: developmental rigor, domain 4: clarity of presentation, domain 5: applicability, and domain 6: editorial independence. The global evaluation of the NOC portrays the general classification and recommendations for use.

This tool was used by the authors to evaluate the extubation protocol formalized by the hospital, entitled "Prevention of unplanned extubation." The following scores were obtained: domain 1 with 85.19\%; domain 2 with $72.22 \%$; domain 3 with $35.42 \%$; domain 4 with $96.30 \%$; domain 5 with $76.39 \%$ and domain 6 with $100 \%$. In the global evaluation of the guideline, the overall quality score was 4 . The guideline analyzed was recommended for use, but with modifications.

\section{Discussion}

Unplanned extubation is a recurrent adverse event in intensive care units, especially in those dedicated to the pediatric specialty. Its occurrence can be prevented using the norms and protocols of the health institutions, and the appropriate guidelines for each situation. The patient safety in PICUs theme comes up since it is the central axis in the well-being, care, and evolution of the patient's clinical condition. Patient safety helps in the tendency to improve the general health condition, especially when associated with the effectiveness in the quality of the service offered.

In the work of Aydon, et al. [10] it was found that male babies have a higher incidence of UE, $n=31$ (75.6\%). Contrary to what was found in this research: among the 22 patients analyzed, 13 are female, which represents $59.1 \%$ of the total sample.

In a systematic review, seven studies identified that restlessness/agitation occurred in $13 \%$ to $89 \%$ of all patients who had unplanned extubation [11]. The study by Hatch, et al. [12], on the other hand, states that the most commonly reported because was displacement of the endotracheal tube due to patient movement (40\%), secondary to agitation. Such data agree with the findings found in this study, since among the causes related to the patient (agitation/secretion), these are present in $20 \%$ of the patients in the cardiopediatric ICU and $27 \%$ of the general and surgical ICU.

The study by Silva, et al. [11] demonstrates that the frequency of unplanned extubations associated with bad fixing varied from $8.5 \%$ to $31 \%$, corroborating with the data found in this study. About 7\% (n=1) and 30\% (n = 3) of the patients admitted to general and surgical ICU and cardiopediatric ICU, respectively, presented with complications involving disconnection of the equipment.

The need for reintubation was a marker strongly present in the observed patients, presenting a high frequency in both study samples. Its presence was noted in $82 \%$ of the general and surgical ICU patients and in $78 \%$ of the cardiopediatric ICU patients. Other works in the literature also present high numbers regarding the need for reintubation, corroborating the study. During the first phase of a PICU study, there were 22 episodes of unplanned extubation in 20 patients. Of the 22 unscheduled extubations, $16(73 \%)$ required reintubation [2].

In the literature, there is no consensus about how much is an acceptable rate of UE. Certain authors try indicating some standard. Merkel, et al. [13] identified that a rate of $<$ 2 UE/100 days was adopted by Vermont Oxford Network's in Controversies in Respiratory Care (2012). However, Rachman, et al. [14] states that $1 \mathrm{UE} / 100$ of MV is considered within the US national standards. Disagreeing with both, Meregalli, et al. [2] report that the rate needs to be even lower; they report that the benchmark should be less than 1 UE/100 days of MV.

The analysis of the data from the medical records collected by the authors found a significantly high rate (7.75 for general and surgical ICU and 4.68 for cardiopediatric ICU). This may indicate a specific situation: events erroneously reported by ICU professionals, and therefore implied failures in the authors' collections.

The possibility of missing data is more likely to have happened, since there is no specific document to report the event of unplanned extubation. Notably, the evolution of the ICU patients' charts was disorganized and lacking information.

Regarding the analysis of the extubation protocol performed using the AGREE II tool, in domain 1, referring to the scope and purpose, three questions define the final evaluation [15]. According to the domain, the evaluated protocol mostly presents the specific objectives and the target population described. However, one of the authors graded the domain with a low score on the topic population, 
justified by the fact that there is no description of the target population.

Next, Domain 2 entitled "Stakeholder Involvement" showed consistency among the evaluators' scores. Some flaws were noticed: absence of detailing the area of expertise of the developers and in the description of the professionals responsible for each type of intervention. Since this is a common adverse event in ICUs, the alignment and the way professionals act are essential for the correct approach to the patient victim of unplanned extubation.

In Domain 3, "Rigor of development", there are greater discrepancies between the evaluators' scores. There is no detailed description of the method used to search for evidence; there is no description of how the team developed the NOC using evidence to support recommendations.

There was also no review before publication and there is no forecast for new updates of the guideline. This was the domain with the lowest percentage among the others evaluated. It presents crucial errors, which could be characterized as inattention on behalf of its creators. Updates are essential in these times, due to the vast amount of information and knowledge shared in real time.

Domain 4 "Clarity of presentation" was rated very highly by the research team, as the guideline provides specific and unambiguous recommendations, different forms of approaches and interventions, concurrently, the key recommendations are easily identified.

Domain 5 "Applicability" draws positive attention, since the hospital's guideline describes the implementations that should be put into practice. Regarding monitoring and/ or auditing, one of the topics of the $5^{\circ}$ domain of AGREE II, the guideline in question explains that the hospital has an adverse event notification form, in addition to forwarding it to the Safety Center of the service in question; however, the guideline lacks information about auditing.

Domain 6, entitled "Editorial independence", was disregarded by the reviewer team.

In the item-funding agency, there is no applicability since the responsible institution is a non-profit entity that invests in health, teaching and research activities. The objective of the protocol is to avoid unplanned extubation and its consequences. Thus, it does not present conflicts of interest.

Finally, the overall assessment of the guideline showed a score of 4 . All are in agreement in the recommendation for use, however with adjustments.
The main limitations of this article are based on the difficulty of collecting data from the research field. Failure in communication with the sectors responsible for collection, with consequent delays for the formulation of the work.

It was possible to ascertain approximately $70 \%$ of the extubation causes, the remaining being of unknown origin, due to the lack of complete and unclear data in the medical charts. Incomplete, disconnected and non-readable information hindered the annotation of the information.

The future perspectives of this work are the further dissemination of unplanned extubation in pediatric ICUs, enrichment of the current literature, greater access to knowledge to raise the awareness of professionals and prevent the occurrence of these incidents in hospital settings.

\section{Conclusion}

When resuming the proposed objectives, was analyzed the incidence of unplanned extubation in the service, finding as a result $7.75 \mathrm{UE} / 100$ days of MV for general and surgical ICU and $4.68 \mathrm{UE} / 100$ days of MV for cardiopediatric ICU.

Concomitantly, the characterization of the most prevalent cases of unplanned extubation, both in the general/surgical ICU and in the cardiopediatric ICU, were female patients, 1 year of age or older, intubated due to a congenital cause, with the need for reintubation after extubation, which occurred for an unknown cause, and which did not continue to death as the outcome.

And, after the analysis of the extubation protocol used in this service, it was possible to conclude that there are limitations to its development rigor, but its use in practice is indicated after the changes, aiming at improving the quality of the service and the care to this population.

It is considered that the objectives of analyzing the incidence of unplanned extubation, and analyzing the extubation protocol of a large pediatric hospital were achieved.

The study demonstrates the need for continued education and the development of a culture of patient safety.

\section{Conflict of Interest}

There is no conflict of Interest.

\section{Funding}

The article was not funded. 


\section{Ethical Approval}

There was approval by the Research Ethics Committee. CAAE: 24675319.0.0000.5580

\section{References}

1. Matsumoto T, Carvalho WBD (2007) Intubação traqueal. J Pediatr 83(2): S83-S90.

2. Meregali C, Barona FAJ, Alessandroa LKMAD, Danziay LKEP, Gustavo E. Debaisi GE, et al. (2013) Impacto de una intervención de mejora de calidad sobre la incidencia de extubaciones no planeadas en una unidad de cuidados intensivos pediátricos. Archivos Argentinos de Pediatria 111(5): 391-397.

3. Galloti RMD (2004) Eventos adversos: o que são?. Rev Assoc Med Bras. 50(2): 114.

4. Carvalho FL, Mezzacappa MA, Calil R; Machado HDC (2010) Incidência e fatores de risco para a extubação acidental em uma unidade de terapia intensiva neonatal. Jornal de Pediatria 86(3): 189-195.

5. Tripathi S, Nunes DJ, Katyal C, Ushay HM (2015) Plan to Have No Unplanned: a collaborative, hospital-based quality-improvement project to reduce the rate of unplanned extubations in the pediatric icu. Respiratory Care 60(8): 1105-1112.

6. Crezee KL, DiGeronimo RJ, Rigby MJ, Carter RC, Patel S, et al. (2017) Reducing Unplanned Extubations in the NICU Following Implementation of a Standardized Approach. Respir Care 62(8): 1030-1035.

7. Torres MIU, Pumarega MTM, Lara NRG, Bonis AM, Garcia MEF, et al. (2014) Frecuencia de extubaciones no programadas en una unidad de cuidados intensivos neonatales. Estudio antes y después. An Pediatria 80(5): 304-309.

8. Silva PSLD, Fonseca MCM (2017) Incidence and Risk Factors for Cardiovascular Collapse After Unplanned Extubations in the Pediatric ICU. Respir Care 62(7): 896903.

9. AGREE Next Steps Consortium (2009) The AGREE II Instrument [versão eletrônica].

10. Aydon L,Zimmer, Sharp M (2018) Reporting the incidence of unplanned extubation in the neonatal intensive care unit. J Paediatr Child Health 54(7): 784-787.

11. Silva PS, Reis ME, Aguiar VE, Fonseca MC (2013) Unplanned extubation in the neonatal ICU: a systematic review, critical appraisal, and evidence-based recommendations. Respir Care 58(7): 1237-1245.

12. Hatch LD, Grubb PH, Markham MH, Scott TA, Walsh WF, et al. (2017) Effect of Anatomical and Developmental Factors on the Risk of Unplanned Extubation in Critically Ill Newborns. Am J Perinatol 34(12): 1234-1240.

13. Merkel L, Beers K, Lewis MM, Stauffer J, Mujsce DJ, et al. (2014) Reducing unplanned extubations in the NICU. Pediatrics 133(5): e1367-72.

14. Rachman BR, Watson R, Woods N, Mink RB (2009) Reducing unplanned extubations in a pediatric intensive care unit: a systematic approach. Int J Pediatr 2009: 820495.

15. Gonçalves CC, Pessoa FML, Ribeiro ER (2019) HighSurveillance Drugs Protocol Evaluation-Management of Patient Safety. IOSR Journal of Pharmacy and Biological Sciences 14(6): 59-65. 